\section{ORIGINAL RESEARCH}

L. Sarro

F. Agosta

E. Canu

N. Riva

A. Prelle

M. Copetti

G. Riccitelli

G. Comi

M. Filippi

\title{
Cognitive Functions and White Matter Tract Damage in Amyotrophic Lateral Sclerosis: A Diffusion Tensor Tractography Study
}

BACKGROUND AND PURPOSE: ALS is predominantly a disease of the motor system, but cognitive and behavioral symptoms also are observed. DT MR imaging is sensitive to microstructural changes occurring in WM tracts of patients with ALS. In this study, we investigated the association between cognitive functions and extramotor WM tract abnormalities in ALS patients.

MATERIALS AND METHODS: DT MR imaging was obtained from 16 nondemented patients with ALS and 15 healthy controls. Patients with ALS underwent a neuropsychologic and behavioral evaluation. DT tractography was used to asses the integrity of the CST, corpus callosum, and the major long-range association tracts. The relationship between DT MR imaging metrics and cognitive functions was tested by using linear model analyses, adjusting for age and clinical disability.

RESULTS: Eleven patients (69\%) scored below the fifth percentile in at least 1 cognitive test, and 2 of them had a mild executive impairment. Performances at tests assessing attention and executive functions correlated with DT MR imaging metrics of the corpus callosum, CST, and long association WM tracts bilaterally, including the cingulum, inferior longitudinal, inferior fronto-occipital, and uncinate fasciculi. Verbal learning and memory test scores were associated with fornix DT MR imaging values, whereas visual-spatial abilities correlated with left uncinate fractional anisotropy.

CONCLUSIONS: WM tract degeneration is associated with neuropsychologic deficits in patients with ALS. DT tractography holds promise to gain insight into the role of the brain WM network abnormalities in the development of cognitive impairment in patients with ALS.

ABBREVIATIONS: ALS = amyotrophic lateral sclerosis; ALSbi = ALS with behavioral impairment; ALSci = ALS with cognitive impairment; ALSFRS-R = ALS Functional Rating Scale-revised; CST = corticospinal tract; $\mathrm{DT}=$ diffusion tensor; $\mathrm{FA}=$ fractional anisotropy; $\mathrm{FMRI}=$ functional $\mathrm{MR}$ imaging; FTD = frontotemporal dementia; $\mathrm{GM}=$ gray matter; IFOF = inferior fronto-occipital fasciculus; ILF = inferior longitudinal fasciculus; $M D=$ mean diffusivity; $\mathrm{P}_{\mathrm{FDR}}=P$ value adjusted for multiple comparisons controlling for the false discovery rate; SLF = superior longitudinal fasciculus; $\mathrm{UMN}=$ upper motor neuron; $\mathrm{WM}=$ white matter

D espite the fact that ALS is predominantly a disease of the motor system, cognitive and behavioral symptoms have been described starting more than a century ago. ${ }^{1}$ ALS cooccurs with FTD in $5 \%-15 \%$ of cases, whereas approximately $35 \%$ of nondemented patients with ALS show a mild-to-moderate cognitive impairment. ${ }^{1}$ The most consistently reported cognitive changes in patients with ALS relate to dysfunction of components of the executive system (eg, verbal fluency, set shifting, cognitive inhibition, and selective attention). ${ }^{1}$ There has been somewhat less consistent evidence for impaired memory and language. ${ }^{1}$ Behavioral impairment also is recognized as another feature of ALS, and up to $63 \%$ of patients with

Received February 9, 2011; accepted after revision March 7

From the Neuroimaging Research Unit (L.S., F.A., E.C., G.R., M.F.) and Department of Neurology (L.S., N.R., G.C., M.F.), Institute of Experimental Neurology, Division of Neuroscience, San Raffaele Scientific Institute, Vita-Salute San Raffaele University, Milan, Italy; Neurologic Unit (A.P.), Azienda Ospedaliera Fatebenefratelli e Oftalmico, Milan, Italy; and Biostatistics Unit (M.C.), IRCCS-Ospedale Casa Sollievo della Sofferenza, San Giovanni Rotondo, Foggia, Italy.

Please address correspondence to Massimo Filippi, PhD, Neuroimaging Research Unit, Institute of Experimental Neurology, Division of Neuroscience, Scientific Institute and University Hospital San Raffaele, Via Olgettina, 60, 20132 Milan, Italy; e-mail: m.filippi@hsr.it

Indicates article with supplemental on-line appendix at www.ajnr.org. http://dx.doi.org/10.3174/ajnr.A2658
ALS are apathetic, irritable, inflexible, restless, or disinhibited. ${ }^{1}$

Cognitive impairment in ALS is owing, at least partially, to the presence of ubiquitinated cytoplasmic inclusions of TAR DNA-binding protein 43 underlying both ALS and some types of FTD. ${ }^{1}$ Neuroimaging studies provided compelling evidence that ALS has a significant extramotor cerebral involvement, regardless of the presence of a frontotemporal syndrome or dementia. ${ }^{2}$ Structural MR imaging showed that GM atrophy in patients with ALS extends beyond the motor cortex to the frontotemporal and parietal regions. ${ }^{2} \mathrm{WM}$ atrophy of the corpus callosum, and frontotemporal and occipital areas, also was found. ${ }^{2}$ Many functional imaging studies have shown a widespread frontotemporal lobe involvement in patients with ALS. $^{2}$

A few studies have combined neuropsychologic and neuroimaging findings in patients with ALS. Imaging correlates of cognitive impairment in ALS include atrophy of the frontal lobe; hypometabolism and reduced perfusion of the frontotemporal cortex and anterior cingulate gyrus; and impaired recruitment of the prefrontal, temporal, and parietal cortices during active cognitive tasks. ${ }^{2}$ The most severe cortical involvement has been observed in patients with clinical features of both ALS and FTD. ${ }^{3}$ Much less is known about the association between WM tract damage and cognitive deficits in ALS 
patients. To our knowledge, only $1 \mathrm{MR}$ imaging study ${ }^{4}$ showed atrophy of the frontotemporal WM in patients with ALS with verbal fluency deficits, in the absence of significant GM atrophy, suggesting that an injury to the frontotemporal association pathways probably contributes to cognitive impairment of these patients.

DT tractography allows in vivo localization and reconstruction of WM tracts based on the diffusion properties of water molecules in the brain tissues. DT tractography is now an established tool for the detection of pathology of the CST in ALS. $^{2}$ Recent studies have shown that DT MR imaging is also sensitive to microstructural changes occurring in the extramotor WM tracts of patients with ALS, such as the uncinate fasciculus. ${ }^{2}$ The correlations between WM tract damage and neuropsychologic measures have not been investigated yet.

Against this background, we used DT tractography to reconstruct specific WM tracts and to investigate the association between cognitive functions and extramotor WM tract abnormalities in nondemented patients with ALS.

\section{Materials and Methods}

The study was conducted with institutional review board approval. Written informed consent was obtained from each participant before study enrollment.

\section{Subjects}

Patients with sporadic ALS were recruited consecutively. Inclusion criteria were as follows: a diagnosis of probable laboratory-supported, probable, or definite ALS according to the El Escorial-revised criteria $^{5}$; no family history of ALS; no significant respiratory failure (forced vital capacity $<70$ ); no clinical diagnosis of $\mathrm{FTD}^{6}$; and righthandedness. Patients were excluded if they had cerebrovascular disorders, history of traumatic brain injury, hydrocephalus, or intracranial mass; other neurologic diseases; psychiatric and major medical disorders; psychotropic medication; or history of substance abuse. ALS disease severity was assessed by the ALSFRS-R questionnaire. ${ }^{7}$ Muscle strength was scored by using the Medical Research Council scale from 0 to 5 (higher score indicating greater muscle strength). ${ }^{8}$ UMN involvement was assessed by totaling the number of pathologic UMN signs on examination (higher score indicating more UMN signs; maximum total score $=16){ }^{9}$

Sixteen sporadic patients with sporadic ALS were included. All patients were taking riluzole. Fifteen sex- and age-matched healthy individuals, with no history of neurologic or psychiatric disorders and a normal neurologic examination, served as controls.

\section{Neuropsychological and Behavioral Evaluation}

Within 48 hours from MR imaging, patients with ALS underwent a neuropsychologic and behavioral evaluation. Cognitive and behavioral assessments were administered by a neuropsychologist who was unaware of the clinical and MR imaging data. The neuropsychologic test battery evaluated global cognition with the Mini-Mental State Examination, ${ }^{10}$ verbal memory and learning with the Rey word test, ${ }^{11}$ visuospatial abilities and spatial memory with the test of copy and recall of Rey's figure, ${ }^{12}$ attention and executive functions with the Trail-Making Test (part A that is dependent primarily on the efficiency of visual scanning and psychomotor speed; part B that requires both visuomotor coordination and executive control; and BA score that is calculated as the difference between performance times of part $\mathrm{B}$ and part $\mathrm{A}$ and is considered a measure of cognitive flexibility, which is relatively independent of manual dexterity), ${ }^{13}$ Stroop test, ${ }^{14}$ Wisconsin Card Sorting Test, ${ }^{15}$ and semantic and phonemic fluency tests. ${ }^{16}$ The Mini-Mental State Examination also was administered to the healthy subjects. For each subject, the scores obtained at each domain of the cognitive assessment were adjusted for age and education and sex, as appropriate, and the performance was evaluated according to the percentile distribution in the normative populations. The fifth percentile was used as cutoff because it reflects an equivalent score of 0 when comparing performances of patients with matched normal subjects. A neurobehavioral interview of both patients and caregivers was performed. Behavioral changes also were assessed by using the Neuropsychiatric Inventory scale. ${ }^{17}$

According to current consensus criteria, ${ }^{18}$ those patients who scored at or below the fifth percentile compared with age- and education-matched normal population on at least 2 distinct cognitive tests sensitive to executive functions were considered to have frontotemporal cognitive impairment (ALSci). A diagnosis of ALSbi was made in the presence of 2 behavioral abnormalities, as supported by at least 2 sources from among patient interview/observation, caregiver report, or Neuropsychiatric Inventory. ${ }^{18}$

\section{MR Imaging Acquisition and Analysis}

Using a 1.5T Avanto system (Siemens, Enlargen, Germany), the following sequences were obtained: dual-echo turbo spin-echo; fluidattenuated inversion recovery; and pulsed-gradient spin-echo echoplanar with diffusion-encoding gradients applied in 12 noncollinear directions. A detailed description of MR image parameter is provided in the Supplemental Material.

MR imaging analysis was performed by a single experienced observer, blinded to clinical and cognitive findings. A detailed description of MR imaging analysis is provided in the Supplemental Material. DT analysis was carried out by using in-house software. ${ }^{19}$ Diffusionweighted images were first corrected for distortion induced by eddy currents. The DT was then estimated by linear regression and MD and FA maps were computed. In addition, axial diffusivity and radical diffusivity maps were calculated. An atlas-based automated approach was used to obtain DT MR imaging-derived metrics of WM tracts, including the CST, corpus callosum, cingulum, SLF, IFOF, ILF, uncinate fasciculus, and fornix. This procedure involves 1) the creation of a reference FA image in the standard space (the FA atlas) by using a group of healthy subjects (reference group);2) the definition of WM tract probability maps on the FA atlas; 3 ) the nonlinear alignment of individual study subjects' MD, FA, axial diffusivity, and radical diffusivity maps to the FA atlas; and 4) the application of WM tract probability maps to the individual subject's images to measure mean tract $\mathrm{MD}, \mathrm{FA}$, axial diffusivity, and radical diffusivity.

\section{Statistical Analysis}

Differences between groups in demographic and clinical variables were assessed by using the Pearson $\chi^{2}$ test or the Mann-Whitney $U$ test, for categoric and continuous variables, respectively. DT MR imaging indices were compared between groups by using multivariate linear models adjusting for subject age. In patients with ALS, the association between neuropsychologic test scores and DT MR imaging metrics was assessed by using multivariate linear models adjusting for subject age and ALSFRS-R. To minimize the number of comparisons, only MD and FA values entered the correlation analysis. Results are reported as beta coefficients along with their $P$ values. Partial and overall $R^{2}$ also were provided. $P$ values also were adjusted for multiple comparisons controlling for the false discovery rate $\left(\mathrm{p}_{\mathrm{FDR}}\right)$. A $P$ value 


\begin{tabular}{|c|c|c|c|}
\hline & $\begin{array}{l}\text { Healthy } \\
\text { Controls }\end{array}$ & ALS Patients & $P^{\mathrm{a}}$ \\
\hline $\bar{N}$ & 15 & 16 & \\
\hline Age (yr) & $63 \pm 12(43-77)$ & $61 \pm 10(40-74)$ & 0.47 \\
\hline Women $(\%)$ & $8(53)$ & $8(50)$ & 0.85 \\
\hline Education (yr) & $12 \pm 6(5-24)$ & $9 \pm 4(5-17)$ & 0.18 \\
\hline Disease duration (mo) & & $29 \pm 19(3-84)$ & \\
\hline ALSFR-R score & & $33 \pm 7(22-44)$ & \\
\hline UL-MRC score & & $54 \pm 11(39-68)$ & \\
\hline LL-MRC score & & $33 \pm 15(2-48)$ & \\
\hline UMN score & & $13 \pm 4(3-16)$ & \\
\hline
\end{tabular}

Note:-Values are means \pm SD (range) or $N$ (percentages); UL-MRC indicates Upper Limb-Medical Research Council; LL-MRC, Lower Limb-Medical Research Council.

a Pearson $\chi^{2}$ test or the Mann-Whitney $U$ test, as appropriate.

$<.05$ was considered as significant. Statistical analysis was performed by using SAS Release 9.1 (SAS Institute, Cary, North Carolina).

\section{Results}

Among the 16 patients with ALS, included in the study, 9 patients had a definite ALS, 4 patients a probable ALS, and 3 patients a probable laboratory-supported ALS. Thirteen patients had a limb-onset, 1 a bulbar-onset, and 2 a limb + bulbar onset. There was no difference in age, sex, and education between ALS patients and controls (Table 1). Controls had a mean MMSE score \pm SD of $28.4 \pm 1.7$ that was not significantly different from that of ALS patients $(P=.61)$. Table 2 shows the neuropsychologic findings in ALS patients. Five patients (31\%) had normal cognitive scores. Eleven patients $(69 \%)$ scored below the cutoff in at least 1 cognitive test, and 2 of them (12.5\%) were classified as ALSci. Patients with ALS impaired in at least 1 cognitive test had greater Upper Limb Medical Research Council scale score compared with those with normal cognitive tests $(P=.003)$; no other demographic and clinical differences were found between patient groups. Two patients $(12.5 \%)$ had a Neuropsychiatric Inventory total domain score $\geq 3$ on at least 2 domains ( 1 patient had aberrant motor behaviors and eating disorders, and 1 patient had mild anxiety, depression, apathy, irritability and sleep behavior disorders). In 1 case, a diagnosis of ALSbi was made; this patient did not have executive impairment.

On the dual-echo and fluid-attenuated inversion recovery scans, hyperintensities of the CST were detected bilaterally in $8(50 \%)$ patients with ALS and 6 (40\%) healthy controls. In patients with ALS, the CST hyperintensities were identified in the caudal portion of the posterior limb of the internal capsule and extended upward through the corona radiata and downward to the ventral portion of the brain stem. In the 6 healthy subjects, the abnormal signal intensity was limited to the internal capsule and did not extend to the corona radiata or to the brain stem. Table 3 shows the means and SDs of DT MR imaging metrics of the WM tracts studied in patients with ALS and healthy controls. Compared with healthy controls, patients with ALS showed increased MD of the corpus callosum $(P<.04)$, CST bilaterally (right, $P=.001$; left, $P=.002$ ), SLF bilaterally $(P=.02$ for both $)$, right cingulum $(P=.02)$, uncinate fasciculus bilaterally ( $P=.02$ for both), and decreased FA of the CST bilaterally (right, $P=.001$; left, $P=.002$ ). Pa-

\begin{tabular}{|c|c|c|}
\hline & $\begin{array}{l}\text { Mean } \pm \text { SD } \\
\text { (Range) }\end{array}$ & $\begin{array}{c}N \text { of Patients } \\
\text { with an Abnormal } \\
\text { Performance (\%) }\end{array}$ \\
\hline \multicolumn{3}{|l|}{ General cognition } \\
\hline MMSE (cutoff, 24) & $28.0 \pm 2.0(24-30)$ & 0 \\
\hline \multicolumn{3}{|l|}{ Verbal learning } \\
\hline $\begin{array}{l}\text { Rey's words, immediate recall } \\
\text { (cutoff, 28.53) }\end{array}$ & $43 \pm 12(18-60)$ & 0 \\
\hline \multicolumn{3}{|l|}{ Verbal memory } \\
\hline $\begin{array}{l}\text { Rey's words, delayed recall } \\
\text { (cutoff, 4.69) }\end{array}$ & $10 \pm 3(5-15)$ & 0 \\
\hline \multicolumn{3}{|l|}{ Spatial memory } \\
\hline $\begin{array}{l}\text { Rey's figure recall (cutoff, } \\
\text { 9.47) }\end{array}$ & $15 \pm 7(8-29)$ & $2(12.5)$ \\
\hline \multicolumn{3}{|l|}{$\begin{array}{l}\text { Attention and executive } \\
\text { functions }\end{array}$} \\
\hline TMT-A (cutoff, 93) & $44 \pm 49(5-191)$ & $1(6)$ \\
\hline TMT-B (cutoff, 282) & $75 \pm 70(4-278)$ & 0 \\
\hline TMT-BA (cutoff, 186) & $32 \pm 25(1-88)$ & 0 \\
\hline Stroop test (cutoff, 36.91) & $32 \pm 18(11-69)$ & $3(19)$ \\
\hline $\begin{array}{l}\text { WCST, global score (cutoff, } \\
\text { 90.50) }\end{array}$ & $66 \pm 29(20-109)$ & $3(19)$ \\
\hline Phonemic fluency (cutoff, 17) & $34 \pm 12(14-55)$ & $2(12.5)$ \\
\hline Semantic fluency (cutoff, 25) & $41 \pm 10(21-59)$ & $1(6)$ \\
\hline \multicolumn{3}{|l|}{ Visuospatial abilities } \\
\hline $\begin{array}{l}\text { Rey's figure copy (cutoff, } \\
\text { 28.88) }\end{array}$ & $26 \pm 6(17-33)$ & $5(31)$ \\
\hline \multicolumn{3}{|l|}{ Behavioral functioning } \\
\hline NPI, global score & $2 \pm 4(0-12)$ & $2(12.5)^{\mathrm{a}}$ \\
\hline
\end{tabular}

Note:-Scores are corrected for age, gender, and education; MMSE indicates Mini-Mental State Examination; TMT, Trail-Making Test; WCST, Wisconsin Card Sorting Test; NPI, Neuropsychiatric Inventory.

Stroop and TMT test scores are expressed in seconds.

aPI total domain score (severity $\times$ frequency) $\geq 3$ on at least 2 domains.

tients with ALS relative to controls also had increased radical diffusivity of the CST bilaterally ( $P<.001$ for both); SLF bilaterally $(P<.03$ for both $)$ and right uncinate fasciculus $(P=.3)$; and increased axial diffusivity of the right SLF $(P=.04)$, right cingulum $(P=.03)$, and uncinate fasciculus bilaterally (right; $P=.03$; left, $P=.002$ ). When patients with ALS scoring below the cutoff in at least 1 cognitive test were contrasted with healthy controls, DT MR imaging abnormalities were similar to those found in the entire group of patients (Table 3 ); on the contrary, the 5 patients with ALS with normal cognitive tests compared with healthy controls showed only significantly increased MD, increased radical diffusivity, and decreased FA of the CST bilaterally, and increased axial diffusivity of the left uncinate (Table 3 ). No difference was found when the 2 patient groups were compared directly.

Table 4 shows the regression coefficients, $P$ values and goodness of fit measures of the relationships of neuropsychologic test scores with WM tract MD and FA values, adjusted for subject age and ALSFRS-R. Only significant results were reported. Scores on the Trail-Making Test were associated with corpus callosum MD and FA values; with right ILF MD, with CST, cingulum, IFOF, and ILF FA values, bilaterally, and with right uncinate FA (Fig). Performance at the Stroop test was associated with corpus callosum, right IFOF, and right ILF FA values. Wisconsin Card Sorting Test scores correlated with the IFOF and ILF MD values, bilaterally, and the right IFOF FA. 
Table 3: DT MR imaging metrics of specific WM tracts in patients with ALS and healthy controls

\begin{tabular}{|c|c|c|c|c|c|c|c|c|c|}
\hline & & \multicolumn{2}{|c|}{ FA } & \multicolumn{2}{|c|}{$\mathrm{MD}\left(\times 10^{-3} \mathrm{~mm}^{2} \mathrm{~s}^{-1}\right)$} & \multicolumn{2}{|c|}{$\operatorname{radD}\left(\times 10^{-3} \mathrm{~mm}^{2} \mathrm{~s}^{-1}\right)$} & \multicolumn{2}{|c|}{$\operatorname{AxD}\left(\times 10^{-3} \mathrm{~mm}^{2} \mathrm{~s}^{-1}\right)$} \\
\hline & & $\mathrm{HC}$ & ALS & $\mathrm{HC}$ & ALS & $\mathrm{HC}$ & ALS & $\mathrm{HC}$ & ALS \\
\hline$\overline{\mathrm{CC}}$ & & $0.62 \pm 0.03$ & $0.61 \pm 0.03$ & $0.76 \pm 0.04$ & $0.79 \pm 0.05^{*} \S$ & $0.44 \pm 0.04$ & $0.47 \pm 0.05 \S$ & $1.40 \pm 0.06$ & $1.44 \pm 0.05$ \\
\hline \multirow[t]{2}{*}{ CST } & $\mathrm{R}$ & $0.67 \pm 0.03$ & $0.63 \pm 0.04^{*} \S \#$ & $0.71 \pm 0.02$ & $0.75 \pm 0.04^{*} \S \#$ & $0.37 \pm 0.02$ & $0.43 \pm 0.04^{* *} \S \#$ & $1.37 \pm 0.07$ & $1.38 \pm 0.05$ \\
\hline & $\mathrm{L}$ & $0.67 \pm 0.03$ & $0.63 \pm 0.03^{*} \S \#$ & $0.71 \pm 0.03$ & $0.76 \pm 0.04^{*} \S \#$ & $0.38 \pm 0.02$ & $0.44 \pm 0.05^{* *} \S \#$ & $1.37 \pm 0.08$ & $1.39 \pm 0.05$ \\
\hline \multirow[t]{2}{*}{ SLF } & $\mathrm{R}$ & $0.49 \pm 0.03$ & $0.47 \pm 0.03$ & $0.73 \pm 0.04$ & $0.77 \pm 0.06^{*} \S$ & $0.51 \pm 0.04$ & $0.55 \pm 0.06^{*}$ & $1.15 \pm 0.06$ & $1.20 \pm 0.07^{*} \S$ \\
\hline & $\mathrm{L}$ & $0.49 \pm 0.03$ & $0.48 \pm 0.03$ & $0.74 \pm 0.03$ & $0.77 \pm 0.05^{*} \S$ & $0.52 \pm 0.04$ & $0.55 \pm 0.05^{*} \S$ & $1.18 \pm 0.05$ & $1.21 \pm 0.06$ \\
\hline \multirow[t]{2}{*}{ Cingulum } & $\mathrm{R}$ & $0.57 \pm 0.04$ & $0.57 \pm 0.03$ & $0.73 \pm 0.03$ & $0.76 \pm 0.05^{*} \S$ & $0.46 \pm 0.04$ & $0.49 \pm 0.05$ & $1.27 \pm 0.06$ & $1.32 \pm 0.06^{*}$ \\
\hline & $\mathrm{L}$ & $0.57 \pm 0.04$ & $0.56 \pm 0.03$ & $0.75 \pm 0.05$ & $0.78 \pm 0.05$ & $0.48 \pm 0.06$ & $0.50 \pm 0.05$ & $1.31 \pm 0.06$ & $1.35 \pm 0.07$ \\
\hline \multirow[t]{2}{*}{ IFOF } & $\mathrm{R}$ & $0.55 \pm 0.03$ & $0.54 \pm 0.03$ & $0.80 \pm 0.04$ & $0.82 \pm 0.06$ & $0.52 \pm 0.05$ & $0.55 \pm 0.05$ & $1.34 \pm 0.05$ & $1.38 \pm 0.80 \S$ \\
\hline & $\mathrm{L}$ & $0.53 \pm 0.04$ & $0.52 \pm 0.02$ & $0.81 \pm 0.05$ & $0.84 \pm 0.06$ & $0.54 \pm 0.05$ & $0.57 \pm 0.05$ & $1.34 \pm 0.06$ & $1.39 \pm 0.083$ \\
\hline \multirow[t]{2}{*}{ ILF } & $\mathrm{R}$ & $0.46 \pm 0.03$ & $0.45 \pm 0.02$ & $0.81 \pm 0.05$ & $0.84 \pm 0.06$ & $0.60 \pm 0.05$ & $0.62 \pm 0.05$ & $1.23 \pm 0.06$ & $1.27 \pm 0.087$ \\
\hline & $\mathrm{L}$ & $0.47 \pm 0.04$ & $0.46 \pm 0.03$ & $0.81 \pm 0.06$ & $0.83 \pm 0.05$ & $0.58 \pm 0.06$ & $0.61 \pm 0.05$ & $1.26 \pm 0.08$ & $1.27 \pm 0.06$ \\
\hline \multirow[t]{2}{*}{ Uncinate } & $\mathrm{R}$ & $0.43 \pm 0.03$ & $0.42 \pm 0.03$ & $0.79 \pm 0.04$ & $0.83 \pm 0.06^{*} \S$ & $0.59 \pm 0.04$ & $0.63 \pm 0.06 * \S$ & $1.20 \pm 0.05$ & $1.25 \pm 0.07^{*} \S$ \\
\hline & $\mathrm{L}$ & $0.41 \pm 0.03$ & $0.42 \pm 0.03$ & $0.82 \pm 0.05$ & $0.86 \pm 0.06^{*} \S$ & $0.62 \pm 0.05$ & $0.65 \pm 0.06$ & $1.20 \pm 0.07$ & $1.29 \pm 0.07^{*} \S$ \\
\hline Fornix & & $0.40 \pm 0.05$ & $0.40 \pm 0.03$ & $1.18 \pm 0.23$ & $1.22 \pm 0.18$ & $0.93 \pm 0.02$ & $0.96 \pm 0.02$ & $1.67 \pm 0.20$ & $1.73 \pm 0.17$ \\
\hline
\end{tabular}

Note:-Values are means \pm SD; HC indicates healthy controls; radD, radial diffusivity; AxD, axial diffusivity; CC, corpus callosum.

${ }^{*} P<0.05$ and ${ }^{* *} P<0.001$ in patients with ALS versus $\mathrm{HC} ; \S P<.05$ in patients with ALS who scored below the cutoff in at least one cognitive test versus $\mathrm{HC}$, and \# $P<.05$ in patients with ALS with normal cognitive tests versus $\mathrm{HC}$, according to the multivariate linear models adjusted for age (see text for details).

Table 4: Regression coefficients, $P$ values, and goodness of fit measures of the relationships of neuropsychologic test scores with WM tract mean diffusivity and FA values, adjusted for subject age and clinical disability

\begin{tabular}{|c|c|c|c|c|c|c|}
\hline Neuropsychologic Test & DT MR Variable & $\beta$ & $P^{\mathrm{a}}$ & $\mathrm{p}_{\mathrm{FDR}}{ }^{\mathrm{b}}$ & $\begin{array}{c}\text { Partial } \\
R^{2}\end{array}$ & $\begin{array}{c}\text { Overall } \\
R^{2}\end{array}$ \\
\hline \multirow[t]{11}{*}{ TMT-A } & CC MD & 0.008 & .03 & .13 & 0.20 & 0.76 \\
\hline & R ILF MD & 0.007 & .05 & .13 & 0.18 & 0.74 \\
\hline & CC FA & -0.013 & .01 & .04 & 0.25 & 0.81 \\
\hline & R CST FA & -0.016 & .02 & .04 & 0.21 & 0.78 \\
\hline & L CSTFA & -0.016 & .02 & .04 & 0.21 & 0.78 \\
\hline & $R$ cingulum FA & -0.011 & .046 & .07 & 0.18 & 0.74 \\
\hline & R IFOF FA & -0.015 & .01 & .04 & 0.26 & 0.83 \\
\hline & L IFOF FA & -0.020 & .003 & .02 & 0.30 & 0.87 \\
\hline & RILFFA & -0.020 & .003 & .02 & 0.30 & 0.86 \\
\hline & LILFFA & -0.015 & .01 & .04 & 0.24 & 0.80 \\
\hline & R uncinate FA & -0.015 & .02 & .04 & 0.22 & 0.78 \\
\hline \multirow[t]{8}{*}{ TMT-B } & CC MD & 0.01 & .04 & .15 & 0.23 & 0.70 \\
\hline & CC FA & -0.017 & .01 & .051 & 0.30 & 0.77 \\
\hline & $R$ cingulum FA & -0.014 & .047 & .07 & 0.22 & 0.69 \\
\hline & $L$ cingulum FA & -0.016 & .04 & .07 & 0.23 & 0.70 \\
\hline & R IFOF FA & -0.021 & .002 & .01 & 0.38 & 0.85 \\
\hline & L IFOF FA & -0.029 & $<.001$ & .001 & 0.46 & 0.93 \\
\hline & RILFFA & -0.023 & .01 & .051 & 0.29 & 0.76 \\
\hline & R uncinate FA & -0.018 & .03 & .07 & 0.25 & 0.72 \\
\hline \multirow[t]{2}{*}{ TMT-BA } & R IFOF FA & -0.029 & .01 & .09 & 0.36 & 0.72 \\
\hline & L IFOFFA & -0.039 & .004 & .049 & 0.43 & 0.79 \\
\hline \multirow[t]{3}{*}{ Stroop test } & CC FA & -0.014 & .03 & .22 & 0.36 & 0.41 \\
\hline & R IFOF FA & -0.011 & .048 & .22 & 0.32 & 0.37 \\
\hline & R ILFFA & -0.018 & .02 & .22 & 0.41 & 0.46 \\
\hline \multirow[t]{5}{*}{ WCST, global score } & R IFOF MD & -0.007 & .03 & .11 & 0.31 & 0.64 \\
\hline & L IFOF MD & -0.007 & .02 & .11 & 0.34 & 0.67 \\
\hline & R ILF MD & -0.007 & .03 & .11 & 0.32 & 0.65 \\
\hline & L ILF MD & -0.008 & .02 & .11 & 0.35 & 0.68 \\
\hline & R IFOF FA & 0.016 & .02 & .32 & 0.46 & 0.53 \\
\hline Phonemic fluency & $L$ cingulum FA & 0.008 & .01 & .20 & 0.36 & 0.47 \\
\hline \multirow[t]{2}{*}{ Rey's words, immediate recall } & Fornix MD & -0.001 & .04 & .32 & 0.16 & 0.67 \\
\hline & Fornix FA & 0.005 & .004 & .055 & 0.27 & 0.77 \\
\hline Rey's words, delayed recall & Fornix MD & -0.002 & .03 & .43 & 0.50 & 0.62 \\
\hline Rey's figure copy & L uncinate FA & 0.005 & .04 & .51 & 0.32 & 0.73 \\
\hline
\end{tabular}

Note:- $\beta$, regression coefficient; partial $R^{2}$, proportion of variability accounted for by the given DT MRI variable; overall $R^{2}$, proportion of variability accounted for by the full statistical model; TMT, Trail-Making Test; WCST, Wisconsin Card Sorting Test; CC, corpos callosum; CST, corticospinal tract.

a $P$ values according to the multivariate linear models, adjusting for subject's age and ALSFRS-r.

b $P$ values also were adjusted for multiple comparisons controlling the false discovery rate.
b

Phonemic fluency test scores were correlated with the left cingulum FA. When adjusting for multiple comparisons, the relationships between the scores on the Trail-Making Test and the corpus callosum, CST, IFOF, and ILF, bilaterally; and right uncinate FA values remained significant. Verbal learning and memory test scores were associated with fornix MD and FA 

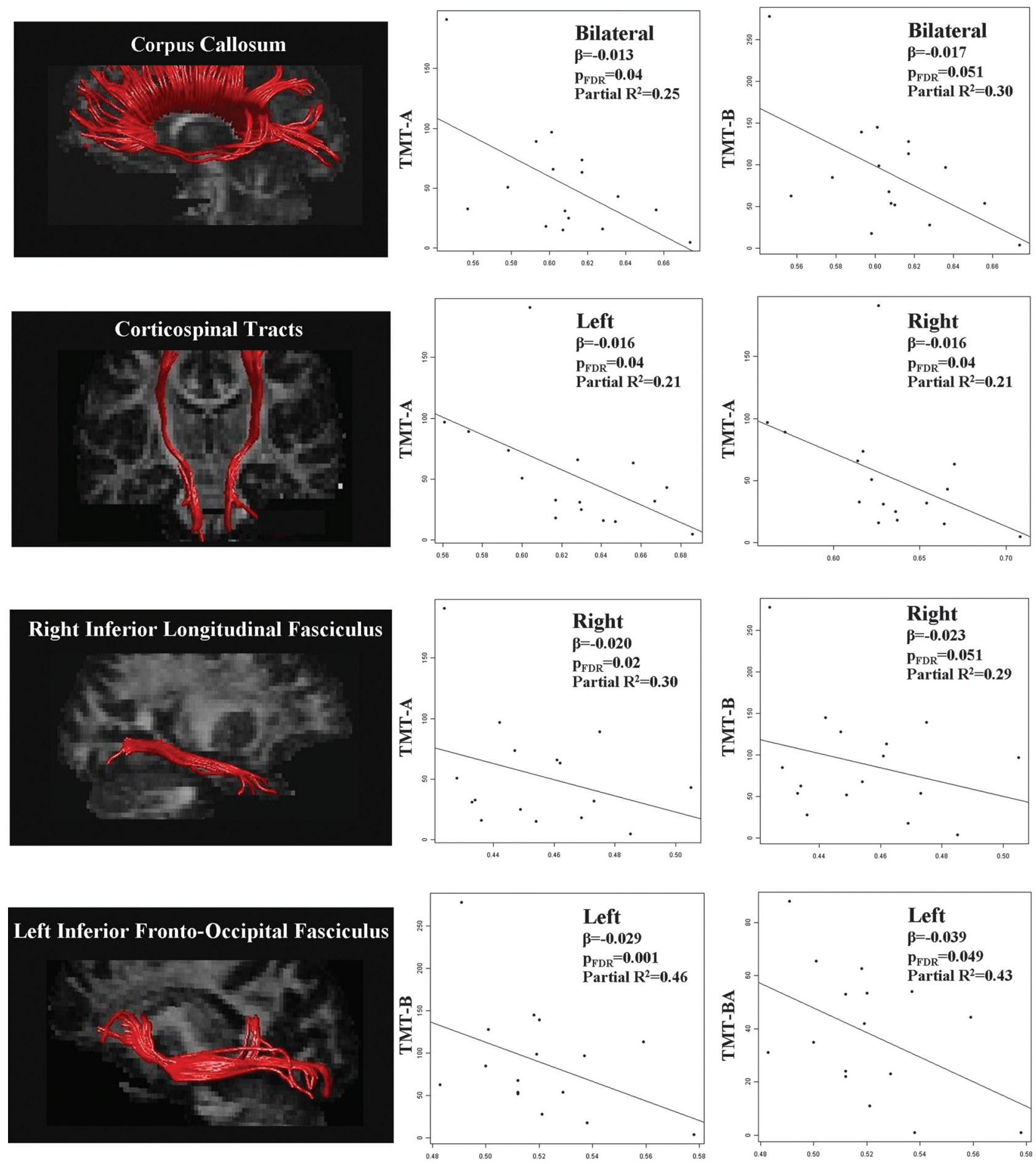

Fig 1. Correlations between performances at the TMT (part A, part B, and difference between performance times of part B and part $A$ ) and FA of the corpus callosum, corticospinal tract, right inferior longitudinal fasciculus, and left inferior fronto-occipital fasciculus in patients with ALS. For illustrative purpose, the left column shows the reconstructions of the WM tracts in a single healthy control (tracts are superimposed onto the mean FA image). The right columns show the scatterplots of the correlations; FA values are reported on the $x$-axis ( $\beta$, regression coefficient; partial $R^{2}$, proportion of variability accounted for by the given DT MR imaging variable); $P_{\mathrm{FDR}}, P$ values adjusted for multiple comparisons controlling for the false discovery rate.

values, whereas visual-spatial abilities correlated with left uncinate FA.

\section{Discussion}

To the best of our knowledge, this is the first study by using DT tractography to investigate the association between microstructural features of WM tracts and neuropsychologic perfor- mance in patients with ALS. In line with previous findings in ALS, ${ }^{1}$ some degree of cognitive involvement was present in $69 \%$ of our nondemented patients with ALS. Overall, the cognitive profile of our sample is consistent with a subtle dysfunction of the executive resources, whereas 2 patients had a mild frontotemporal syndrome according to current consensus criteria. ${ }^{18}$ Some patients also showed spatial short-term memory 
and visuospatial ability deficits. We found that patients with ALS scoring below the cutoff on at least 1 cognitive test showed more widespread extramotor WM damage relative to those patients with normal cognitive tests. The performances at executive function tests correlated with the DT MR imaging measures of corpus callosum and major corticocortical association tracts passing through the frontal and temporal regions, bilaterally. In addition, diffusivity abnormalities of the fornix and the left uncinate were associated with poorer performances at memory and visuospatial tests, respectively. All these findings support a previous voxel-based morphometry study showing that patients wit ALS with evidence of executive and memory dysfunctions had WM volume loss in extramotor regions, which probably belong to the long association bundles connecting the frontal and temporal lobes to other cortical regions. ${ }^{4}$

Performance at the Trail-Making Test, which assesses cognitive flexibility, selective attention, visual scanning, and visual-motor tracking, ${ }^{13}$ was associated with DT MR imaging measures of the corpus callosum, and the long-range frontotemporal (ie, cingulum and uncinate), fronto-occipital (ie, IFOF), and temporo-occipital (ie, ILF) bundles. Most of these associations were evident also when correcting for multiple comparisons. DT MR imaging measures of the corpus callosum and right IFOF and ILF were associated with the performance at the Stroop test, which assesses selective attention and inhibition. Lower FA values of the fronto-occipital and temporo-occipital connections, bilaterally, also were correlated with worse performance at the Wisconsin Card Scoring Test, which is an established measure of rule shifting and mental flexibility. ${ }^{15}$ Previous fMRI studies of healthy subjects demonstrated that the performance at tests assessing executive functions relies on the communication among many broadly distributed, functionally specialized regions of the frontal, temporal, and parietal lobes. ${ }^{20}$ Interestingly, among these cortical regions are those identified by functional imaging studies to be altered in patients with ALS with cognitive impairment. $^{21,22}$ Our results suggest that the integrity of WM tracts that connect these regions is probably a critical factor in executive function impairment in patients with ALS. It is also worth noting that the performance at the simple sequencing subtask of the Trail-Making Test (ie, part A), but not the setshifting subtask (ie, part B), was associated with the CST damage. This is not unexpected, because part A requires the subject to connect consecutive numbers on a paper and seems to be dependent primarily on psychomotor speed. ${ }^{13}$

Impaired verbal fluency, which has been reported in almost all studies of cognitive impairment in ALS, ${ }^{1}$ is thought to be a reflection of working memory and executive functioning deficits rather than of a simple word retrieval impairment. ${ }^{23}$ In our patients, phonemic fluency correlated with left cingulum FA. It has been reported that patients with verbal fluency deficits experience significantly reduced activations of the anterior cingulate cortex, middle and inferior frontal gyri, and parietal and temporal lobes relative to controls during a letter fluency fMRI task. ${ }^{24}$ Besides the association of the cingulate cortices, cingulum fibers connect the middorsolateral frontal cortex with retrosplenial and parietal cortices, thalamus, hippocampus, and parahippocampal gyrus, and they have been suggested to be involved in executive functioning. ${ }^{25}$
With respect to memory performance, the association between verbal learning and memory test scores and fornix MD and FA values is well established and has been documented in DT MR imaging studies of patients with other neurodegenerative and psychiatric diseases. ${ }^{26,27}$ On the contrary, the relationship between visuospatial abilities and left uncinate FA is unexpected and should be interpreted with caution. Although the functional role of the uncinate is still a matter of debate, it probably mediates emotion and memory functions and is part of a ventral language pathway. ${ }^{28}$

Our study is not without limitations. First, the relatively small size of our patient group calls for studies in larger samples. Second, performance scores were not adjusted to take account for motor or speech impairment that may impact on word generation output, and the development of respiratory weakness also has not been accounted for. However, patients with ALS included were all mildly disabled (ALSFRS-R $\geq 22$ ), patients with cognitive impairment had less severe motor disturbances (ie, greater Upper Limb-Medical Research Council score) compared with those with normal cognitive tests, patients with respiratory failure were not included, and the correlation analysis between cognitive and DT MR imaging variables was adjusted for clinical disability. As a consequence, we believe that physical disability may have not influenced the results of our study at a great deal. Third, neuropsychologic tests were not performed in healthy controls.

\section{Conclusions}

This study shows that DT tractography holds promise to gain additional insight into the role of the brain WM network abnormalities to the development of cognitive impairment in patients with ALS. The pattern of cognitive impairment in nondemented patients with ALS is consistent with the extramotor frontotemporal WM damage revealed in the present study. In addition, the finding of significant correlations between the microstructural features of interhemispheric and long-range corticocortical association tracts with performance at tests of executive function and memory suggests that WM tract degeneration may underlie cognitive abnormalities in ALS. Clearly, cognitive deterioration often implies a balance between tissue damage and compensatory mechanisms by the same or other brain systems. As a consequence, our result cannot be interpreted as proof of a direct causal link. Future longitudinal studies are needed to confirm this hypothesis and clarify whether DT MR imaging can serve as a useful tool to predict those patients with ALS who will progress to develop dementia.

\footnotetext{
Disclosures: Federica Agosta, Speaker Bureau: Bayer Schering; Giancarlo Comi, Consultant: Bayer Schering, Serono Symposia International Foundation, Merck Serono International, Sanofi-Aventis, Biogen Dompè, Novartis, and TEVA Pharmaceutical Ind. Details: Honorarium for participation in advisory board and speaking activities; Massimo Filippi, Research Support (including provision of equipment or materials): Bayer-Schering, Biogen-Dompé AG, Genmab A/S, Merck Serono, Teva Pharmaceutical Industries Ltd., Fondazione Italiana Sclerosi Multipla (FISM), and Fondazione Mariani. Speaker Bureau: Bayer Schering Pharma, Biogen-Dompé AG, Genmab A/S, Merck Serono, Teva Pharmaceutical Industries. Consultant: Bayer Schering Pharma, Biogen-Dompé AG, Genmab A/S, Merck Serono, Pepgen Corporation, and Teva Pharmaceutical Industries Ltd.
} 


\section{References}

1. Phukan J, Pender NP, Hardiman O. Cognitive impairment in amyotrophic lateral sclerosis. Lancet Neurol 2007;6:994-1003

2. Agosta F, Chio A, Cosottini M, et al. The present and the future of neuroimaging in amyotrophic lateral sclerosis. AJNR Am J Neuroradiol 2010;31:1769-77

3. Chang JL, Lomen-Hoerth C, Murphy J, et al. A voxel-based morphometry study of patterns of brain atrophy in ALS and ALS/FTLD. Neurology 2005;65: 75-80

4. Abrahams S, Goldstein LH, Suckling J, et al. Frontotemporal white matter changes in amyotrophic lateral sclerosis. J Neurol 2005;252:321-31

5. Brooks BR. El Escorial World Federation of Neurology criteria for the diagnosis of amyotrophic lateral sclerosis. Subcommittee on Motor Neuron Diseases/Amyotrophic Lateral Sclerosis of the World Federation of Neurology Research Group on Neuromuscular Diseases and the El Escorial "Clinical limits of amyotrophic lateral sclerosis" workshop contributors. J Neurol Sci 1994;124 Suppl:96-107

6. Neary D, Snowden JS, Gustafson L, et al. Frontotemporal lobar degeneration: a consensus on clinical diagnostic criteria. Neurology 1998;51:1546-54

7. Cedarbaum JM, Stambler N, Malta E, et al. The ALSFRS-R: a revised ALS functional rating scale that incorporates assessments of respiratory function. BDNF ALS Study Group (Phase III). J Neurol Sci 1999;169:13-21

8. de Carvalho M, Scotto M, Lopes A, et al. Clinical and neurophysiological evaluation of progression in amyotrophic lateral sclerosis. Muscle Nerve 2003;28: $630-33$

9. Turner MR, Cagnin A, Turkheimer FE, et al. Evidence of widespread cerebral microglial activation in amyotrophic lateral sclerosis: an [11C](R)-PK11195 positron emission tomography study. Neurobiol Dis 2004;15:601-09

10. Folstein MF, Folstein SE, McHugh PR. "Mini-mental state". A practical method for grading the cognitive state of patients for the clinician. J Psychiatr Res 1975;12:189-98

11. Carlesimo GA, Caltagirone C, Gainotti G. The Mental Deterioration Battery: normative data, diagnostic reliability and qualitative analyses of cognitive impairment. The Group for the Standardization of the Mental Deterioration Battery. Eur Neurol 1996;36:378-84

12. Caffarra P, Vezzadini G, Dieci F, et al. Rey-Osterrieth complex figure: normative values in an Italian population sample. Neurol Sci 2002;22:443-47

13. Giovagnoli AR, Del Pesce M, Mascheroni S, et al. Trail making test: normative values from 287 normal adult controls. Ital J Neurol Sci 1996;17:305-09

14. Barbarotto R, Laiacona M, Frosio R, et al. A normative study on visual reaction times and two Stroop colour-word tests. Ital J Neurol Sci 1998;19:161-70
15. Laiacona M, Inzaghi MG, De Tanti A, et al. Wisconsin card sorting test: a new global score, with Italian norms, and its relationship with the Weigl sorting test. Neurol Sci 2000;21:279-91

16. Novelli G, Papagno C, Capitani E, et al. Tre test clinici di ricerca e produzione lessicale. Taratura su soggetti normali. Arch Psicol Neurol Psichiatr 1986;47: 477-506

17. Cummings JL, Mega M, Gray K, et al. The Neuropsychiatric Inventory: comprehensive assessment of psychopathology in dementia. Neurology 1994;44: 2308-14

18. Strong MJ, Grace GM, Freedman M, et al. Consensus criteria for the diagnosis of frontotemporal cognitive and behavioural syndromes in amyotrophic lateral sclerosis. Amyotroph Lateral Scler 2009;10:131-46

19. Filippi $M$, Cercignani $M$, Inglese $M$, et al. Diffusion tensor magnetic resonance imaging in multiple sclerosis. Neurology 2001;56:304-11

20. Zakzanis KK, Mraz R, Graham SJ. An fMRI study of the Trail Making Test. Neuropsychologia 2005;43:1878-86

21. Kew JJ, Goldstein LH, Leigh PN, et al. The relationship between abnormalities of cognitive function and cerebral activation in amyotrophic lateral sclerosis. A neuropsychological and positron emission tomography study. Brain 1993; 116(Pt 6):1399-423

22. Abrahams S, Goldstein LH, Kew JJ, et al. Frontal lobe dysfunction in amyotrophic lateral sclerosis. A PET study. Brain 1996;119(Pt 6):2105-20

23. Abrahams S, Leigh PN, Harvey A, et al. Verbal fluency and executive dysfunction in amyotrophic lateral sclerosis (ALS). Neuropsychologia 2000;38: $734-47$

24. Abrahams S, Goldstein LH, Simmons A, et al. Word retrieval in amyotrophic lateral sclerosis: a functional magnetic resonance imaging study. Brain 2004; 127:1507-17

25. Schermuly I, Fellgiebel A, Wagner S, et al. Association between cingulum bundle structure and cognitive performance: an observational study in major depression. Eur Psychiatry 25:355-60

26. Sexton CE, Mackay CE, Lonie JA, et al. MRI correlates of episodic memory in Alzheimer's disease, mild cognitive impairment, and healthy aging. Psychiatry Res 184:57-62

27. Nestor PG, Kubicki M, Kuroki N, et al. Episodic memory and neuroimaging of hippocampus and fornix in chronic schizophrenia. Psychiatry Res 2007;155: 21-28

28. Parker GJ, Luzzi S, Alexander DC, et al. Lateralization of ventral and dorsal auditory-language pathways in the human brain. Neuroimage 2005;24: $656-66$ 